\title{
Dynamic leakage current compensation in ferroelectric thin-film capacitor structures
}

\author{
René Meyer ${ }^{\mathrm{a})}$ and Rainer Waser \\ CNI, Center of Nanoelectronic Systems for Information Technology (CNI), Forschungszentrum Jülich, \\ 52425 Jülich, Germany
}

Klaus Prume, Torsten Schmitz, and Stephan Tiedke

Aixacct Systems, 52068 Aachen, Germany

(Received 6 December 2004; accepted 17 February 2005; published online 1 April 2005)

\begin{abstract}
We report on a measurement procedure to separate ferroelectric switching current and dielectric displacement current from the leakage current in leaky ferroelectric thin-film capacitor structures. The ac current response is determined for two adjacent frequencies. Taking advantage of the different frequency dependencies of the ferroelectric switching current, dielectric displacement current and ohmic current, the hysteresis loop is calculated without performing a static leakage current measurement, which causes a high dc field stress to the sample. The applicability of the proposed measurement procedure is demonstrated on a $\mathrm{Pt} / \mathrm{Pb}(\mathrm{Zr}, \mathrm{Ti}) \mathrm{O}_{3} / \mathrm{IrO}_{2}$ ferroelectric capacitor revealing a high leakage current. (C) 2005 American Institute of Physics. [DOI: 10.1063/1.1897425]
\end{abstract}

Over the past 15 years, ferroelectrics such as $\mathrm{PbZr}_{x} \mathrm{Ti}_{1-x} \mathrm{O}_{3}(\mathrm{PZT})$ or $\mathrm{SrBi}_{2} \mathrm{Ta}_{2} \mathrm{O}_{9}$ (SBT) have been intensively studied in view of their suitability as dielectrics for future nonvolatile high integrated random access memory thin-film capacitors (FeRAM). ${ }^{1}$ Key material parameters for memory applications are the remnant polarization $P_{R}$ and the coercive voltage $V_{C}$ or the coercive field $E_{C}$. Since the remnant polarization directly correlates with the amount of charge that can be detected during the read operation of the memory cell, high values are desired for memory applications. The coercive voltage is closely linked with the operating voltage of the storage element. In an ideal system, $V_{C}$ determines the voltage that is needed to switch the polarization inside the ferroelectric thin film. In real materials, the operating voltage might be twice the coercive voltage, since a voltage higher than $V_{C}$ is required to switch the polarization completely and a low $\kappa$ interface layer may reduce the field inside the ferroelectric layer. ${ }^{2}$ Both values can be obtained from the hysteresis curve $(P-V$ characteristic) of the ferroelectric capacitor. For a loss-free ferroelectric capacitor, the hysteresis loop can be acquired by an integration over the displacement current passing through the capacitor. In contrast, real ferroelectric capacitors show a leakage current in addition that superposes the displacement current and that can affect the shape of the hysteresis curve. ${ }^{3}$ An integration of the monitored current may then result in a nonphysical increase of remnant polarization and coercive field.

Graphical $^{4}$ as well as numerical methods ${ }^{5}$ have been reported to eliminate the leakage contribution from the current response. A common method is to monitor and to withdraw the static leakage current from the dynamic current measurement. ${ }^{5}$ Here, the voltage is changed stepwise and the dc current is recorded. This measurement procedure has to be repeated for each data point, whereby the duration of a voltage step mainly depends on the relaxation time of the transient signal response and on the required resolution of the monitored current. A typical duration for a voltage step is in the order of seconds. Depending on the number of data

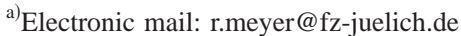

points, several minutes are required to measure the dc $I-V$ characteristic. In some cases, the sample cannot withstand the continuous dc field stress, and the characteristic of the capacitor will change during the static leakage current measurement, e.g., due to resistance degradation. Since this tendency increases with reduced sample thickness, the characterization of ultrathin ferroelectric films is a tough business. In view of the continuous trend to miniaturization, an increase of leakage current originating from a contamination of the edges of the cell capacitor during the processing ${ }^{6,7}$ might also complicate the material characterization.

We propose a measurement principle, further denoted as dynamic leakage current compensation (DLCC) for leakage current compensation without dc stressing the sample. The key idea concerns the utilization of different frequency dependencies of the dielectric, the ferroelectric and the ohmic contribution to the total current that allows an elimination of the leakage current on the basis of two ac current measurements performed at adjacent frequencies. First, the principle of DLCC will be discussed for a ferroelectric model capacitor. Subsequently, the concept will be applied to real data obtained for a PZT capacitor. At a given frequency, the electrical behavior of the ferroelectric capacitor might be represented by an equivalent electric circuit consisting of a parallel association of a passive resistive and a passive capacitive element in parallel with a time invariant voltage controlled charge source serving for the displacement current in case of ferroelectric switching. In good approximation, we assume the resistive element $R$ only to depend on the applied voltage $V_{\text {ext }}$. A significant frequency dependence $f(\omega)$ is not considered. $R$ is then given by

$$
R=f\left(V_{\text {ext }}\right) \neq f(\omega)
$$

and the current through the resistor $i_{R}$ amounts to

$$
i_{R}=\frac{V_{\mathrm{ext}}}{R} \neq f(\omega) .
$$

The capacitive element $C$ might also show a voltage dependence given by 


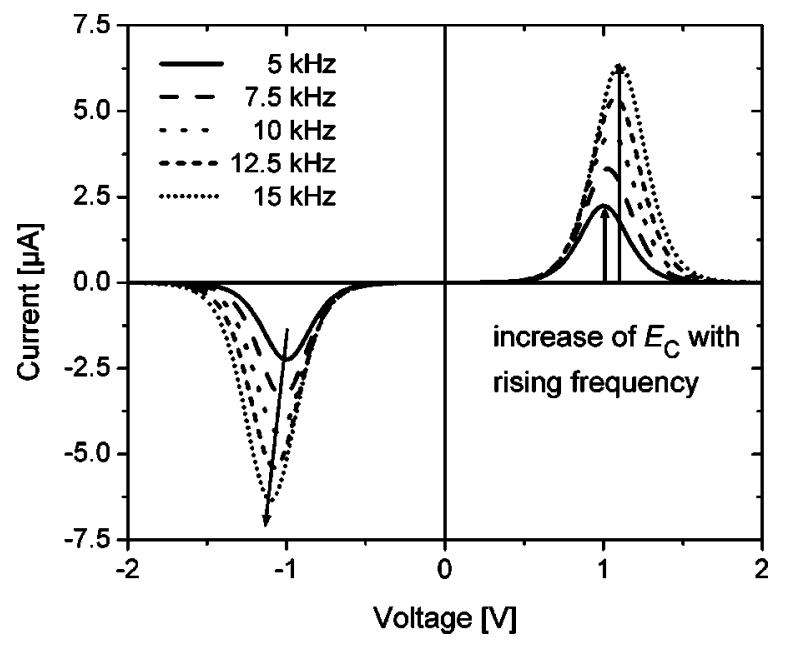

FIG. 1. Frequency dependent current response of an idealized ferroelectric capacitor (simulation). Since the time integral over the current peak corresponds to the polarization charge $P_{S}$ and the time window, in which the applied voltage is sufficiently high to switch the polarization (here between 0.5 and $1.5 \mathrm{~V}$ ), reciprocally scales with the frequency, the switching current has to increase linearly with frequency. Furthermore, a slight increase of $E_{C}$ with frequency is found experimentally.

$$
C=f\left(V_{\text {ext }}\right) .
$$

The current passing through the capacitive element can then be calculated to

$$
i_{C}=j \omega C \times V_{\mathrm{ext}} \propto \omega .
$$

To estimate the frequency dependence of the current initiated by switching the ferroelectric polarization, we assume that by sweeping the external voltage from $V_{\min }$ at $t_{0}$ to $V_{\max }$ at $t_{1}$, the spontaneous polarization $P_{S}$ of the ferroelectric will switch completely. Then, $P_{S}$ might be given by

$$
P_{S}=\frac{1}{A} \int_{t_{0}}^{t_{1}} i_{F} d \tau,
$$

whereby $i_{F}$ is the ferroelectric displacement current caused by a switching of the spontaneous polarization and $A$ denotes the area of the capacitor. Since the time window $t_{1}-t_{0}$ reciprocally scales with the frequency $\omega$ of the voltage source, the switching current has to increase with frequency to keep $P_{S}$ constant. The relation between $\omega$ and $i_{F}$ is illustrated in Fig. 1. If the shape of the ferroelectric current contribution does not depend on frequency, the ferroelectric switching current reveals a similar frequency dependence as the dielectric displacement current:

$$
i_{F} \propto \omega
$$

In real ferroelectric thin-film capacitors a weak increase of $V_{C}$ with frequency has been observed. ${ }^{8-10}$ Thus, Eq. (6) has to be regarded as a first order approximation, where the frequency dependence of $V_{C}$ is neglected.

The total current $i$ through the capacitor is given by the sum of the individual component currents:

$$
i=i_{R}+i_{C}(\omega)+i_{F}(\omega) .
$$

In case of a linear frequency dependence of $i_{C}$ and $i_{F}, i_{C}(\omega)$ might be replaced by $i_{C}^{0} \times \omega$ and $i_{F}(\omega)$ by $i_{F}^{0} \times \omega$. Then, the current $i$ amounts to reversal, a strong current increase is found at higher voltages.
Downloaded 21 Dec 2006 to 134.94.122.39. Redistribution subject to AIP license or copyright, see http://apl.aip.org/apl/copyright.jsp

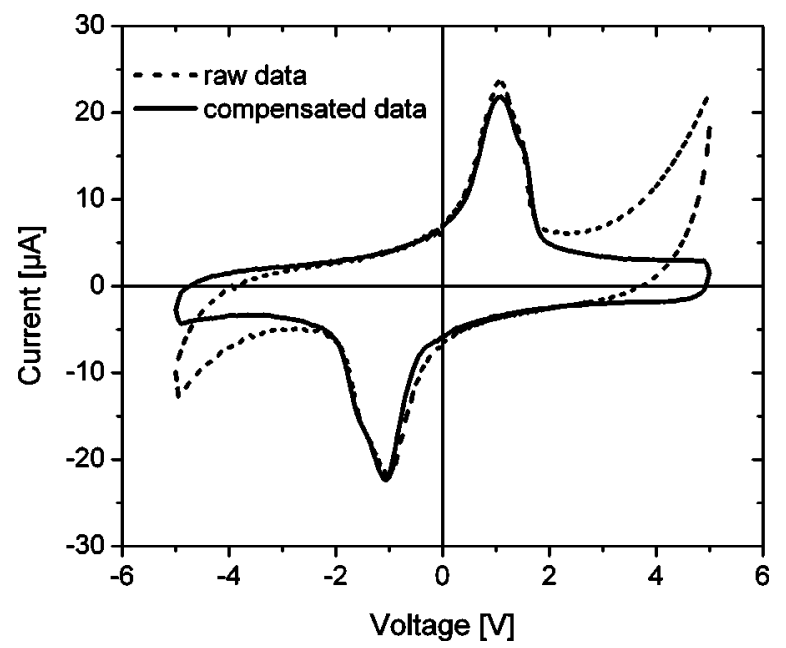

FIG. 2. Current response in reply to a triangle voltage excitation of a leaky ferroelectric thin-film capacitor (dashed line) at $100 \mathrm{~Hz}$ and compensated current response calculated from the difference of two $I-V$ curves obtained at 100 and $200 \mathrm{~Hz}$ (full line).

$$
i=i_{R}+\omega i_{C}^{0}+\omega i_{F}^{0} .
$$

Let us now define $\Delta i$ as the difference between two currents $i\left(\omega_{2}\right)-i\left(\omega_{1}\right)$ obtained for two frequencies $\omega_{1}$ and $\omega_{2}$, whereby we assume $\omega_{2}>\omega_{1}$. Since the resistive element is assumed to be frequency independent, we obtain a quantity that contains only the capacitive and the ferroelectric contribution of the signal:

$$
\Delta i=\left(\omega_{2}-\omega_{1}\right)\left(i_{C}^{0}+i_{F}^{0}\right) .
$$

For $\omega_{2}=2 \omega_{1}$, Eq. (9) simplifies to

$$
\Delta i=\left(\omega_{1}\right)\left(i_{C}^{0}+i_{F}^{0}\right) \equiv \omega_{1} i_{C}+\omega_{1} i_{F} \equiv i_{\text {comp }}\left(\omega_{1}\right) .
$$

Here, $i_{\text {comp }}\left(\omega_{1}\right)$ denotes the current response that might be obtained for a leakage free capacitor at the frequency $\omega_{1}$. For the practical application, both frequencies should be chosen close enough to fulfill the assumption made in Eqs. (1) and (6). For any frequency $\omega$, the leakage current free current response is calculated to

$$
i_{\text {comp }}(\omega)=\frac{\omega}{\omega_{2}-\omega_{1}}\left[i\left(\omega_{2}\right)-i\left(\omega_{1}\right)\right]
$$

A further limitation in the choice of both frequencies has to be seen in the signal to noise ratio. Good results are obtained for $1.2<\omega_{2} / \omega_{1}<2$. For $\omega_{1} \rightarrow 0$, we find the static leakage compensation as shown in Ref. 5.

To confirm the proposed procedure, measurements are carried out on a $\mathrm{Pt} / \mathrm{PZT} / \mathrm{IrO}_{2}$ capacitor structure. In three coating steps, PZT films of $150 \mathrm{~nm}$ thickness were crystallized on a platinized silicon substrate by a chemical solution deposition method. After annealing at $700{ }^{\circ} \mathrm{C}$ for $20 \mathrm{~min}$, $\mathrm{IrO}_{2}$ top electrodes of $50 \mathrm{~nm}$ thickness were deposited. Finally, a postannealing step of $700{ }^{\circ} \mathrm{C}$ for 5 min was performed. Electrical characterizations in virtual ground mode are performed by the TF ANALYZER 2000 FE (aixACCT Systems). Measurements with triangular waveform and $5 \mathrm{~V}$ peak to peak voltage are performed at different frequencies $\omega_{1}=100 \mathrm{~Hz}$ and $\omega_{1}=200 \mathrm{~Hz}$. Results at $100 \mathrm{~Hz}$ are illustrated in Fig. 2 (dashed line). Beside two current peaks at $+1 \mathrm{~V}$ and $-1 \mathrm{~V}$ that can be attributed to the polarization 


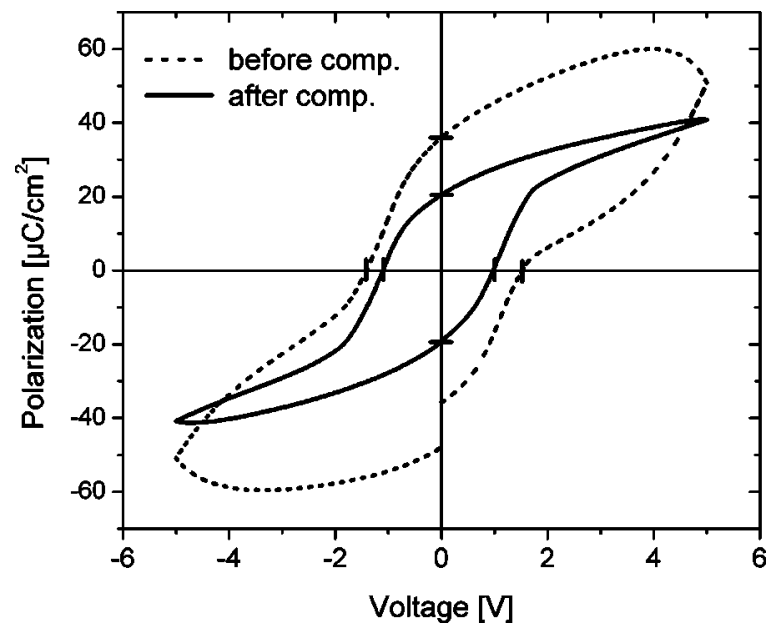

FIG. 3. Uncompensated and dynamic leakage current compensated hysteresis curve of a leaky ferroelectric thin-film capacitor. As shown, both remnant polarization and coercive voltage may not reflect the real ferroelectric behavior of the material in the presence of leakage currents.

The respective hysteresis curve calculated from the uncompensated current response is shown in Fig. 3 (dashed line). By current integration, we obtain a typical hysteresis curve for a leaky ferroelectric capacitor. In contrast, the compensated current response does not obey the current increase at higher voltages observed for the uncompensated data (see Fig. 2, solid line). An integration of the compensated current is shown in Fig. 3 (solid line). Obviously, the presence of a leakage current strongly effects both remnant polarization and coercive field. As illustrated in Fig. 3, remnant polarization and coercive field seem to be increased in the uncompensated $P-V$ curve, which is of course a nonphysical interpretation.

A further conspicuous feature of the proposed method is that the compensated hysteresis loop is closed. In contrast, the hysteresis loop calculated from raw data shows a significant gap. We like to mention that no additional data manipulation was carried out except the above given procedure. We conclude that for the investigated capacitors, the unclosed hysteresis loop is a leakage current related and not a retention related phenomenon (relaxation of $P_{R}$ ). A reasonable explanation for this unexpected result might be as follows: Due to an asymmetry in the contact properties, the leakage current might depend on the polarity of the applied voltage. This approach is supported by the raw data shown in Fig. 2. An integration of the current over one cycling period will then give a quantity nonequal to zero, which directly correlates to the size of the gap. The proposed method might therefore be even more interesting, if ferroelectric capacitors with different electrode materials will be studied. ${ }^{11}$

Summarizing our results, the present contribution addresses the problematic of leakage current and leakage current compensation in ferroelectric thin-film capacitor structures. It is shown that an ohmic contribution in the current response of a leaky ferroelectric capacitor has a significant impact on the shape of the hysteresis curve and lead to nonphysical values of the remnant polarization and the coercive field. It is demonstrated that the proposed measurement procedure is an efficient method to drastically reduce the influence of the leakage current without performing a static leakage current measurement. Simultaneously, short measurement periods compared to the static leakage compensation method enable more efficient device testing and reduce the dc field stress of the sample. This might improve the certainty of hysteresis data, if the electrical properties are affected by a static monitoring of leakage current, e.g., by resistance degradation. The concept is also applicable, if the leakage current depends on the polarity of the applied voltage, which might originate from different work functions of top and bottom electrode.

The authors are indebted to S. Halder for fruitful discussions.

${ }^{1}$ J. F. Scott, The Physics of Ferroelectric Ceramic Thin Films for Memory Application, Ferroelectric Review (Grodon and Breach, Amsterdam, 1998).

${ }^{2}$ M. Grossmann, O. Lohse, D. Bolten, U. Böttger, and R. Waser, J. Appl. Phys. 92, 2688 (2002).

${ }^{3}$ H. N. Lee, D. Hesse, N. Zakharov, and U. Gösele, Science 296, 2006 (2002).

${ }^{4}$ C. Xiaogang, Y. Xi, Z. Desheng, and Z. Liangying, Ferroelectrics 259, 55 (2001).

${ }^{5}$ Operation Manual of the TF Analyzer 2000, Aixacct Systems GmbH, Germany.

${ }^{6}$ W. Hartner, G. Schindler, V. Weinrich, M. Ahlstedt, H. Schroeder, R. Waser, C. Dehm, and C. Mazure, Integr. Ferroelectr. 27, 1257 (1999).

${ }^{7}$ G. J. M. Dormans, P. K. Larsen, G. A. C. M. Spierings, J. Dikken, M. J. E. Ulenaers, R. Cuppens, D. J. Taylor, and R. D. J. Verhaar, Integr. Ferroelectr. 6, 93 (1995).

${ }^{8}$ J. F. Scott, Integr. Ferroelectr. 12, 71 (1996).

${ }^{9}$ O. Lohse, M. Grossmann, U. Böttger, D. Bolten, and R. Waser, J. Appl. Phys. 89, 2332 (2002).

${ }^{10}$ L. W. Chen and Y. Wang, Appl. Phys. Lett. 75, 4186 (1999).

${ }^{11}$ J. Lee, C. H. Choi, B. H. Park, T. W. Noh, and J. K. Lee, Appl. Phys. Lett. 72, 3380 (1998) 